\title{
Efficacy of teriparatide in the treatment of nontraumatic osteonecrosis of the femoral head: a retrospective comparative study with alendronate
}

Ryuta Arai ${ }^{1 *}$ D, Daisuke Takahashi ${ }^{1}$, Masahiro Inoue ${ }^{2}$, Tohru Irie ${ }^{1}$, Tsuyoshi Asano ${ }^{1}$, Takuya Konno ${ }^{1}$, Mohamad Alaa Terkawi ${ }^{1}$, Tomohiro Onodera', Eiji Kondo ${ }^{3}$ and Norimasa Iwasaki ${ }^{1}$

\begin{abstract}
Background: Collapse of the femoral head associated with nontraumatic osteonecrosis (NOFH) is one of the most common causes of disability in young adult patients. Excessive bone resorption by osteoclast coincident with the suppression of osteogenesis are believed to be responsible for collapse progression. Alendronate that inhibits bone resorption by inducing osteoclast apoptosis has been traditionally used for treating NOFH; however, several reports documented serious complications by the use of this drug. On the other hand, teriparatide activates osteoblasts leading to an overall increase in bone volume, and is expected to reduce the progression of femoral head collapse in NOFH. Therefore, the present study was undertaken to examine pharmacological effects of teriparatide on collapse progression of NOFH and to compare these effects with alendronate.
\end{abstract}

Methods: We conducted a retrospective study in our facility for comparing the pharmacological effects of teriparatide and alendronate on 32 NOFH patients diagnosed with osteoporosis. Between 2007 and 2013, patients were treated with daily administration of $20 \mu \mathrm{g}$ teriparatide (15 patients: $18 \mathrm{hips}$ ), or with $35 \mathrm{mg}$ of alendronate once a week (17 patients: 22 hips). The mean period of follow-up was 18.7 months. The progression of collapse was evaluated prior to the administration and later every three months by anteroposterior radiographs. Collapse progression with $>1 \mathrm{~mm}$ was defined as advanced collapse, while with $<1 \mathrm{~mm}$ was defined as stable radiologic disease. Student's t-test and the chi-square test was used to do compare the pharmacological effects of the two groups.

Results: Treatment with terparatide had a tendency to reduce the rate of advanced collapse as compared to that with alendronate $(p=0.105)$. Kaplan-Meier curves related to stable radiologic disease showed that teriparatide-treated patients had better stable states than these treated with alendronate ( $p=0.08$, log-rank test). Moreover, treatment with teriparatide resulted in a significant reduction in collapse progression as compared to that with alendronate, noted at the end of follow-up period $(p=0.049)$.

Conclusion: The present study suggests that teriparatide has greater pharmacological effects than alendronate for treating NOFH and preventing the collapse of femoral head.

Trial registration: The registration number in UMIN Clinical Trial Registry is UMIN000017582. The date of registration is May 5, 2015.

Keywords: Nontraumatic osteonecrosis of the femoral head, Teriparatide, Collapse of the femoral head

\footnotetext{
* Correspondence: ryutaaraisti@yahoo.co.jp

'Department of Orthopaedic Surgery, Hokkaido University Graduate School

of Medicine, Kita-15, Nish-7, Kita-ku, Sapporo 060-8638, Japan

Full list of author information is available at the end of the article
}

(c) The Author(s). 2017 Open Access This article is distributed under the terms of the Creative Commons Attribution 4.0 International License (http://creativecommons.org/licenses/by/4.0/, which permits unrestricted use, distribution, and reproduction in any medium, provided you give appropriate credit to the original author(s) and the source, provide a link to the Creative Commons license, and indicate if changes were made. The Creative Commons Public Domain Dedication waiver (http://creativecommons.org/publicdomain/zero/1.0/) applies to the data made available in this article, unless otherwise stated. 


\section{Background}

Complete collapse of the femoral head is one of the major complication of nontraumatic osteonecrosis $(\mathrm{NOFH})$, resulting in dysfunction of the hip and disability in patients. Collapse of the femoral head occurs in $75 \%$ of NOFH cases within three years and in $80 \%$ of patients within four years of onset of hip pain $[1,2]$. The disease is typically progressive and mainly occurs in young population, whereas and most of patients eventually require total hip replacement (THR) within three years [3-5]. In fact, $10 \%$ of THRs performed in the United States is due to NOFH [4]. The pathogenesis of NOFH remains unclear, but involves interruption of the blood supply to the femoral head leading to osteonecrosis [5]. Consequently, osteonecrosis induces osteoclastic and osteoblastic remodeling processes that cause collapse of the femoral head of the necrotic region [6-8].

Efficient pharmacological treatment that prevents bone collapse is not currently available, and therefore, identifying novel drug with potential benefits is extremely desirable. Coordinating osteoclastic and osteoblastic activities is an attractive approach and may offer good therapeutic option for NOFH. Bisphosphonates that inhibit excessive osteoclast-mediated bone resorption have proven to reduce the incidence of collapse of femoral head in osteonecrotic hip [9-11]. Nonetheless, Chen et al reported that alendronate has only minor effects on the disease progression and has failed to prevent THR in majority of patients [12]. On the other hand, teriparatide is a recombinant form of parathyroid hormone that has been used as anabolic agent for treatment of osteoporosis. Teriparatide has positive effects on osteoblast differentiation and activation leading to bone necrosis lesions repair [13-15]. In support of this concept, Jiang et al reported that teriparatide improves trabecular morphology and increases cancellous bone volume and cortical bone thickness $[16,17]$. Teriparatide enhances the bone healing in osteonecrotic jaw, and reduces steroid-induced osteonecrosis of femoral head [18-21].

Although several clinical studies highlight the advantageous effects of terpiaratide for the treatment of osteonecrosis, the clinical benefit of teriparatide for $\mathrm{NOFH}$ has not been systematically studied. Therefore, the present study was undertaken to determine whether teriparatide could offer greater pharmacological effects than alendronate, which is known to be the traditional pharmacological option for preventing collapse progression of the femoral head.

\section{Methods}

\section{Patients}

Between Jan 1, 2007, and Dec 31, 2013, fifty-three patients who were diagnosed with NOFH had undergone treatment for osteoporosis; nineteen patients had received teriparatide, and thirty-four patients had received alendronate. Twenty-one patients (four patients on teriparatide treatment and seventeen patients on alendronate treatment) were excluded from this study; fifteen patients had been followed for less than 6 months. Four patients developed osteoarthritis of the hip. In two patients, the administration periods were not identified. We conducted retrospective study for these thirty-two patients. Fifteen patients (18 hips; three patients were observed in bilateral hips) received teriparatide, and seventeen patients (22 hips; five patients were observed in bilateral hips) received alendronate. The patients' mean age was 38.7 years, with 4 male and 11 female patients, in the teriparatide group, and 46.8 years, with 9 male and 8 female patients, in the alendronate group (Table 1). The mean follow-up was 18.7 months. The radiologic stages of the patients were stages 1,2 , and $3 \mathrm{~A}$ in the Japanese Investigation Committee (JIC) staging system, and the locations of osteonecrosis were type $\mathrm{C}-1$ and $\mathrm{C}-2$ in the JIC classification system [22]. Stage 1 is the phase that osteonecrosis can be detected by magnetic resonance imaging (MRI) or bone scintigram, cannot be detected by $\mathrm{X}$-ray. In stage 2, demarcating sclerosis is seen without collapse of femoral head on X-ray images. Stage 3 shows collapse of the femoral head without joint-space narrowing, and is subdivided into stage 3A (less than $3 \mathrm{~mm}$ of collapse) and $3 \mathrm{~B}$ (3 $\mathrm{mm}$ or more of collapse). Type $C$ lesion occupies more than the medial two-thirds of the weight-bearing portion. Type C-2 lesion extends laterally to the acetabular edge, whereas type C-1 lesion does not [22].

Table 1 Demographic characteristics of the teriparatide and alendronate groups

\begin{tabular}{llll}
\hline & $\begin{array}{l}\text { Teriparatide } \\
\text { group }\end{array}$ & $\begin{array}{l}\text { Alendronate } \\
\text { group }\end{array}$ & p value \\
\hline Patients & 15 & 17 & \\
Hips & 18 & 22 & 0.053 \\
Mean age, years (range) & $38.7(22-59)$ & $46.8(19-67)$ & 0.499 \\
Mean body weight (kg) & 59.2 & 59.2 & 0.127 \\
Mean body mass index (kg/m²) & 22.9 & 21.5 & 0.071 \\
Mean follow up, days (range) & $523.7(217-719)$ & $606.9(336-724)$ & \\
Radiologic stage in JIC & & & \\
1 & $3(16.7 \%)$ & $0(0 \%)$ & \\
2 & $8(44.4 \%)$ & $17(77.3 \%)$ & \\
3A & $7(38.9 \%)$ & $5(22.7 \%)$ & \\
Locations of osteonecrosis in JIC & & & \\
Type C-1 & $8(44.4 \%)$ & $8(36.4 \%)$ & \\
Type C-2 & $10(55.6 \%)$ & $14(63.6 \%)$ & \\
\hline
\end{tabular}

JIC Japanese Investigation Committee 


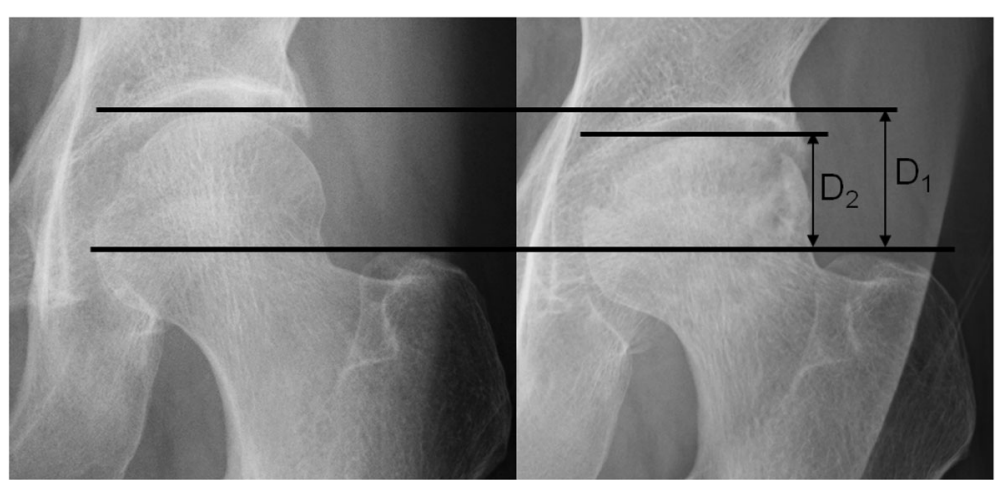

Fig. 1 The progression of collapse of the femoral head of NOFH. The progression of collapse $\left(D_{1}-D_{2}\right)$ before administration (left) and at every follow-up (right) using anteroposterior radiographs. The baseline is the top of the greater trochanter of the femur

\section{Treatments}

Teriparatide $(20 \mu \mathrm{g})$ was subcutaneously administered once per day $[23,24]$ while alendronate $(35 \mathrm{mg})$ was orally administered once per week [25]. In Japan, daily $20 \mu \mathrm{g}$ teriparatide and weekly $35 \mathrm{mg}$ alendronate administration is approved for the treatment of osteoporosis. None of the patients received vitamin D3, and non-steroid anti-inflammatory agents were given when needed to relief the pain.

\section{Assessment of collapse progression}

Plain anteroposterior hip radiographs were taken prior to the treatment courses and at every interval of between three to six-month follow-up visit. The JIC staging and locations of osteonecrosis were estimated on X-ray examinations or MRI. Collapse progression of the femoral head was evaluated on anteroposterior hip radiographs in neutral rotation before administration and at every follow-up (Fig. 1) with the same imaging conditions, for avoidance of differences caused by magnification effects. Collapse progression with $>1 \mathrm{~mm}$ was defined as "advanced collapse", and as "stable radiologic disease" when collapse progression was $<1 \mathrm{~mm}$. The follow-up periods for the treatment courses were assigned for two years.

\section{Statistical analysis}

Student's $t$-test and the chi-square test was used to compare the pharmacological effects of the two groups. Normality of the samples were confirmed by Shapiro-Wilk test. Stable radiologic disease analysis was performed using Kaplan-Meier analysis. Inter-group comparison of Kaplan-Meier data was performed using the log-rank test. A $p$-value $<0.05$ was deemed significant, as described in the figure legends. Analyses were performed using Ekuseru-Toukei 2010 (Social Survey Research Information Co., Ltd., Tokyo, Japan).

\section{Results}

To investigate the pharmacological usefulness of teriparatide for treatment of $\mathrm{NOFH}$, a 6-months to 2-years follow-up study of 15 patients (18hips) who received teriparatide was performed. In parallel, same follow-up study of 17 patients (22 hips) who received alendronate was performed to compare the efficiencies of teriparatide. There were no complications observed on the patients during the period of study caused by the treatment course of teriparatide or alendronate. Notably, collapse progression was observed in $59.1 \%$ of patients received alendronate treatment, and in $33.3 \%$ of patients received teriparatide treatment, suggesting that teriparatide had a tendency to reduce collapse progression of femoral head (Table 2). Next, Kaplan-Meier curves were generated for patients with advanced collapse as the end-point (Fig. 2). Although there was no significant difference between the two treatments, patients treated by teriparatide exhibited a 6-month-prolonged radiologic disease $(94.4 \%(90 \%$ CI $85.6-100))$ as compared to alendronate $(77.3 \%$ (90\% CI 62.6 - 92.0)). Moreover, a longer 1-year stability (83.3\% (90\% CI 68.9 - 97.8)) was noted in patients treated by teriparatide than that noted in patients treated by alendronate $(53.6 \%$ (90\% CI 35.8 - 71.3) ( $p=0.08$, log-rank test). Moreover, treatment with teriparatide resulted in a significant reduction in collapse progression $(0.67 \mathrm{~mm})$ as compared to that with alendronate (1.24 mm; $p=0.049$ ) (Table 2). Representative case 1 is a

Table 2 The occurrence rate of advanced collapse in the teriparatide and alendronate groups at the end of follow-up and the final collapse progression in the teriparatide and alendronate groups

\begin{tabular}{llll}
\hline & Teriparatide group & Alendronate group & $p$ value \\
\hline Advanced collapse & $6 / 18(33.3 \%)$ & $13 / 22(59.1 \%)$ & 0.105 \\
Final collapse & $0.67 \mathrm{~mm}$ & $1.24 \mathrm{~mm}$ & $0.049^{\ddagger}$ \\
progression & $(0.00-3.61 \mathrm{~mm})$ & $(0.00-3.22 \mathrm{~mm})$ & \\
\hline
\end{tabular}

¥Significant 


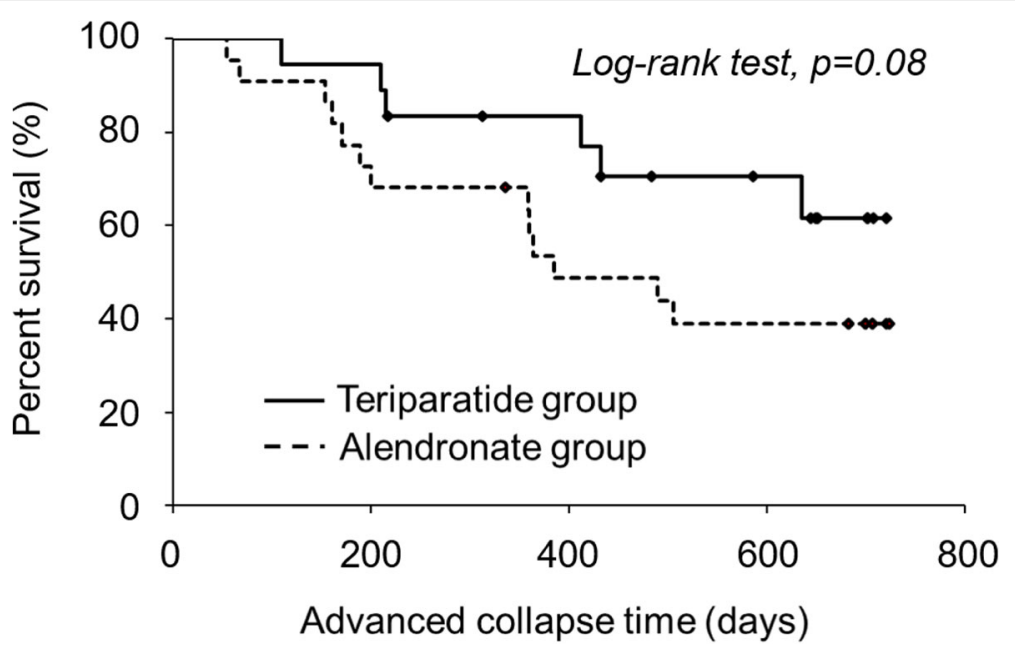

Fig. 2 The Kaplan-Meier curves of the teriparatide group (solid line) and the alendronate group (dotted line) with advanced collapse as the end-point

67-year-old man with NOFH, type C-2 in the JIC classification and JIC stage 2 that was treated by alendronate (Fig. 3). Collapse progression was $3.3 \mathrm{~mm}$ after 21 months of treatment course, and eventually THR was performed (Fig. 3). Representative case 2 is a 27-year-old woman with NOFH, type C-2 in the JIC classification and JIC stage 1 that was treated by teriparatide (Fig. 4). Collapse progression of the femoral head did not occur over a period of 20 months (Fig. 4). Taken together, our data suggest that teriparatide is promising therapeutic agent for NOFH.

\section{Discussion}

NOFH causes decreased vascular supply to the trabecular bone of the femoral head, resulting in collapse of articular surface of the femoral head and severe hip pain in young adults. The precise mechanism of collapse in $\mathrm{NOFH}$ has not been clarified; however, excessive bone resorption by osteoclast in necrotic regions is thought to induce collapse of the femoral head [7]. The disease is progressive and once collapse of the femoral head occurs, osteoarthritis of the hip joint is developed in few years. Most of cases need THR for relieving the severe hip pain caused by osteoarthritis; however, due to limited durability of THR, this procedure is not recommended for young adults. Therefore, conservative treatment that prevents collapse progression of the femoral head in early stage of NOFH is highly demanded.

Several pharmacological agents have been used for treatment of $\mathrm{NOFH}$, including statins [26], anticoagulants [27, 28], prostacyclin [29, 30], and bisphosphonates. Bisphosphonates, namely alendronate, are the most common used [31]. Alendronate prevents early collapse of the femoral head in NOFH [32], and improves clinical function with better rate of collapse [11]. However, the frequently reported failures of alendronate in preventing collapse progression and its serious complications including osteonecrosis and atypical fractures [12, 33-38] highlight the need for more effective and safer therapeutic option for the treatment of NOFH.

We speculate that enhancing osteogenesis might contribute to the prevention of femoral head collapse progression. Teriparatide is known to increase the
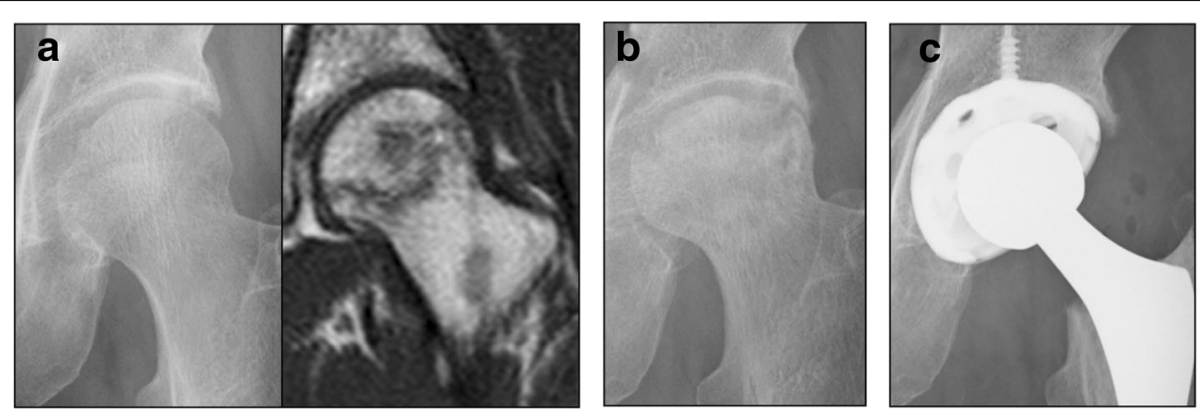

Fig. 3 Case 1. Sixty-seven years old man with NOFH. a Anteroposterior radiograph and magnetic resonance imaging (T1WI) at the first examination showing NOFH. After diagnosis, alendronate was administered. b Collapse of the femoral head has progressed. $\mathbf{c}$ Eventually, THR was performed 


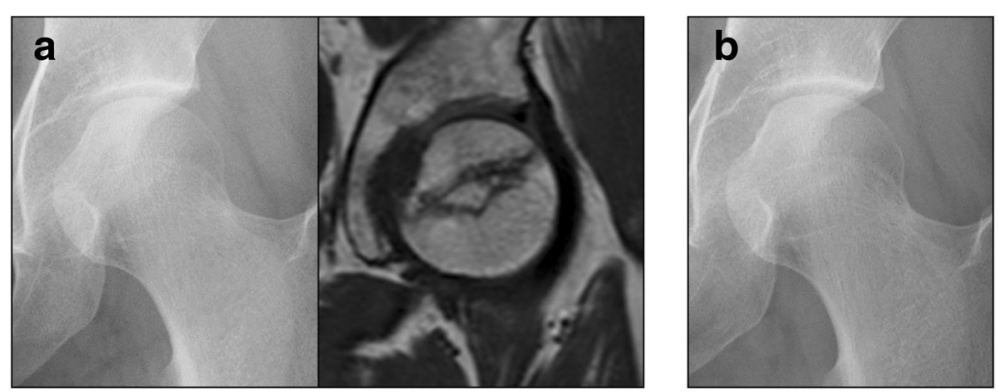

Fig. 4 Case 2. Twenty-seven years old woman with NOFH. a Anteroposterior radiograph and magnetic resonance imaging (T1WI) at the first examination showing NOFH. After diagnosis, teriparatide was administered. $\mathbf{b}$ The femoral head showed no progression of collapse for one year and 8 months

life-span of mature osteoblasts by preventing their apoptosis [39]. Teriparatide has been reported to increase cancellous bone volume and connectivity, and to improve trabecular morphology with a shift toward more plate-like structure [18]. Moreover, intermittent use of teriparatide exerts anabolic action on cortical bone with improved cancellous bone microarchitectures [40]. Recently, several case reports showed successful outcome of teriparatide for the treatment of osteonecrosis [18-20] and fracture-healing [41]. Consistently, our study showed that treatment with teriparatide resulted in lower rate of collapse progression than alendronate. Treatment with alendronate has been reported to be correlated with high incidence of collapse progression (65.6\%) [12]. Likewise, in our study, the rate of head collapse in the alendronatetreated group fell within this range (59.1\%), while lesser incidence was noted in teriparatide-treated patients $(33.3 \%)$. This can be explained by the fact that teriparatide enhances osteoblast activity in the necrotic lesion, leading to an increase in cancellous bone volume and trabecular thickness of the femoral head.

This study has some limitations that must be pointed out. The mean follow-up period was longer in the alendronate group, and the patient's average age of teriparatide group was 8 years younger than alendronate group. This may give an advantage of bone growth. The current study is retrospective study, covers small sample size (number of patients), doesn't include the clinical outcome or functional parameters post treatments, and doesn't consider the differences between radiological stage or location of osteonecrosis in the patients. Further study, including a larger number of samples with longer follow-up period is needed to conclusively demonstrate the therapeutic use of teriparatide for treatment of NOFH.

\section{Conclusion}

This is the first study demonstrating that teriparatide is a potent conservative option for treatment of NOFH. The treatment course by teriparatide results in lesser collapse progression of the femoral head than that by alendronate, which is considered as the traditional pharmacological option for preventing collapse progression of the femoral head.

\section{Abbreviations}

JIC: Japanese Investigation Committee; NOFH: Nontraumatic osteonecrosis of the femoral head; THR: Total hip replacement

\section{Acknowledgements \\ None.}

\section{Funding}

No specific funding was received from any funding bodies in the public, commercial or not-for-profit sectors to carry out the work described in this article.

\section{Availability of data and materials}

All data supporting our findings are contained within the manuscript.

\section{Authors' contributions}

RA was involved in the design of the study, performed the clinical assessment, analysis and interpretation of data, and drafted and revised the manuscript. DT, MI, TI, TA, TK, MAT, TO, was involved in the design of the study, assisted with data interpretation, and revised the manuscript for important intellectual content. EK, NI was involved in the design of the study and the data acquisition and revised the manuscript critically for important intellectual content. All authors have read and approved the final manuscript.

\section{Competing interests}

The authors declare that they have no competing interests. The authors do not have any commercial or collaborative relationships that could be constructed as biased or inappropriate. The decision to submit this paper for publication was not influenced by any funding body.

\section{Consent for publication}

Not applicable.

\section{Ethics approval and consent to participate}

This study was approved by the Institutional Review Board (IRB) of Hokkaido University Hospital (approval ID: 014-0327), and registered in UMIN Clinical Trial Registration (Registration number: UMIN000017582). Informed consent was not obtained from the participants due to the IRB provision for the retrospective study.

\section{Author details}

${ }^{1}$ Department of Orthopaedic Surgery, Hokkaido University Graduate School of Medicine, Kita-15, Nish-7, Kita-ku, Sapporo 060-8638, Japan. ²Department of Orthopaedic Surgery, Wajo Eniwa Hospital, Koganechuo 2-1-1, Eniwa 061-1149, Japan. ${ }^{3}$ Department of Advanced Therapeutic Research for Sports Medicine, Hokkaido University Graduate School of Medicine, Kita-15, Nish-7, Kita-ku, Sapporo 060-8638, Japan. 
Received: 6 July 2016 Accepted: 22 December 2016 Published online: 19 January 2017

\section{References}

1. Merle D'Aubigné R, Postel M, Mazabraud A, Massias P, Gueguen J, France P. Idiopathic necrosis of the femoral head in adults. J Bone Joint Surg Br. 1965:47:612-33.

2. Ohzono K, Saito M, Sugano N, Takaoka K, Ono K. The fate of nontraumatic avascular necrosis of the femoral head. A radiologic classification to formulate prognosis. Clin Orthop Relat Res. 1992;277:73-8.

3. Aaron RK, Lennox D, Bunce GE, Ebert T. The conservative treatment of osteonecrosis of the femoral head. A comparison of core decompression and pulsing electromagnetic fields. Clin Orthop Relat Res. 1989;249:209-18.

4. Mont MA, Jones LC, Sotereanos DG, Amstutz HC, Hungerford DS. Understanding and treating osteonecrosis of the femoral head. Instr Course Lect. 2000;49:169-85.

5. Mont MA, Hungerford DS. Non-traumatic avascular necrosis of the femoral head. J Bone Joint Surg Am. 1995;77:459-74.

6. Kenzora JE, Steele RE, Yosipovitch ZH, Glimcher MJ. Experimental osteonecrosis of the femoral head in adult rabbits. Clin Orthop Relat Res. 1978;130:8-46

7. Wang C, Wang X, Xu XL, Yuan XL, Gou WL, Wang AY, Guo QY, Peng J, Lu SB. Bone microstructure and regional distribution of osteoblast and osteoclast activity in the osteonecrotic femoral head. PLoS One. 2014;9:e96361.

8. Shibayama K. Idiopathic osteonecrosis of the femoral head in the elderly. Kurume Med J. 2000;47:37-43.

9. Agarwala S, Sule A, Pai BU, Joshi VR. Study of alendronate in avascular necrosis of bone. J Assoc Physicians India. 2001:49:949-50.

10. Nishii T, Sugano N, Miki H, Hashimoto J, Yoshikawa H. Does alendronate prevent collapse in osteonecrosis of the femoral head? Clin Orthop Relat Res. 2006;443:273-9.

11. Agarwala S, Shah S, Joshi VR. The use of alendronate in the treatment of avascular necrosis of the femoral head: follow-up to eight years. J Bone Joint Surg Br. 2009;91:1013-8.

12. Chen $\mathrm{CH}$, Chang JK, Lai KA, Hou SM, Chang CH, Wang GJ. Alendronate in the prevention of collapse of the femoral head in nontraumatic osteonecrosis: a two-year multicenter, prospective, randomized, doubleblind, placebo-controlled study. Arthritis Rheum. 2012;64:1572-8.

13. Niall HD, Sauer RT, Jacobs JW, Keutmann HT, Segre GV, O'Riordan JL, Aurbach GD, Potts Jr JT. The amino-acid sequence of the aminoterminal 37 residues of human parathyroid hormone. Proc Natl Acad Sci U S A. 1974;71:384-8.

14. Dobnig H, Turner RT. The effects of programmed administration of human parathyroid hormone fragment (1-34) on bone histomorphometry and serum chemistry in rats. Endocrinology. 1997;138:4607-12.

15. Shen V, Dempster DW, Birchman R, Xu R, Lindsay R. Loss of cancellous bone mass and connectivity in ovariectomized rats can be restored by combined treatment with parathyroid hormone and estradiol. J Clin Invest. 1993;91:2479-87.

16. Mosekilde L, Søgaard CH, Danielsen CC, Tørring O. The anabolic effects of human parathyroid hormone ( $\mathrm{hPTH}$ ) on rat vertebral body mass are also reflected in the quality of bone, assessed by biomechanical testing: a comparison study between hPTH-(1-34) and hPTH-(1-84). Endocrinology. 1991;129:421-8

17. Jiang Y, Zhao JJ, Mitlak BH, Wang O, Genant HK, Eriksen EF. Recombinant human parathyroid hormone (1-34) [teriparatide] improves both cortical and cancellous bone structure. J Bone Miner Res. 2003;18:1932-41.

18. Harper RP, Fung E. Resolution of bisphosphonate-associated osteonecrosis of the mandible: possible application for intermittent low-dose parathyroid hormone [rhPTH(1-34)]. J Oral Maxillofac Surg. 2007;65:573-80.

19. Lau AN, Adachi JD. Resolution of osteonecrosis of the jaw after teriparatide [recombinant human PTH-(1-34)] therapy. J Rheumatol. 2009;36:1835-7.

20. Lee JJ, Cheng SJ, Jeng JH, Chiang CP, Lau HP, Kok SH. Successful treatment of advanced bisphosphonate-related osteonecrosis of the mandible with adjunctive teriparatide therapy. Head Neck. 2011;33:1366-71.

21. Dong Y, Li Y, Huang C, Gao K, Weng X. Systemic application of teriparatide for steroid induced osteonecrosis in a rat model. BMC Musculoskelet Disord. 2015;16:163

22. Sugano N, Atsumi T, Ohzono K, Kubo T, Hotokebuchi T, Takaoka K. The 2001 revised criteria for diagnosis, classification, and staging of idiopathic osteonecrosis of the femoral head. J Orthop Sci. 2002;7:601-5.
23. Miyauchi A, Matsumoto T, Sugimoto T, Tsujimoto M, Warner MR, Nakamura T. Effects of teriparatide on bone mineral density and bone turnover markers in Japanese subjects with osteoporosis at high risk of fracture in a 24-month clinical study: 12-month, randomized, placebo-controlled, double-blind and 12-month open-label phases. Bone. 2010;47:493-502.

24. Tsujimoto $M$, Uenaka $K$, Iwata A, Higashiuchi $Y$, Sowa $H$. Effects of teriparatide in Japanese and non-Japanese populations: bridging findings on pharmacokinetics and efficacy. J Bone Miner Metab. 2012;30:326-37.

25. Uchida S, Taniguchi T, Shimizu T, Kakikawa T, Okuyama K, Okaniwa M, et al. Therapeutic effects of alendronate $35 \mathrm{mg}$ once weekly and $5 \mathrm{mg}$ once daily in Japanese patients with osteoporosis: a double-blind, randomized study J Bone Miner Metab. 2005;23(5):382-8.

26. Pritchett JW. Statin therapy decreases the risk of osteonecrosis in patients receiving steroids. Clin Orthop Relat Res. 2001;386:173-8.

27. Glueck CJ, Freiberg RA, Sieve L, Wang P. Enoxaparin prevents progression of stages I and II osteonecrosis of the hip. Clin Orthop Relat Res. 2005;435:164-70.

28. Wang Y, Yin L, Li Y, Liu P, Cui Q. Preventive effects of puerarin on alcoholinduced osteonecrosis. Clin Orthop Relat Res. 2008;466:1059-67.

29. Disch AC, Matziolis G, Perka C. The management of necrosis-associated and idiopathic bone-marrow oedema of the proximal femur by intravenous iloprost. J Bone Joint Surg Br. 2005;87:560-4.

30. Jäger M, Tillmann FP, Thornhill TS, Mahmoudi M, Blondin D, Hetzel GR, et al. Rationale for prostaglandin 12 in bone marrow oedema-from theory to application. Arthritis Res Ther. 2008;10:R120.

31. Green J, Clézardin P. The molecular basis of bisphosphonate activity: a preclinical perspective. Semin Oncol. 2010;37:S3-11.

32. Lai KA, Shen WJ, Yang CY, Shao CJ, Hsu JT, Lin RM. The use of alendronate to prevent early collapse of the femoral head in patients with nontraumatic osteonecrosis. A randomized clinical study. J Bone Joint Surg Am. 2005:87:2155-9.

33. Wong PK, Borromeo GL, Wark JD. Bisphosphonate-related osteonecrosis of the jaw in non-malignant bone disease. Rheumatol Int. 2013;33:2189-98.

34. Conte Neto N, Spolidorio LC, Andrade CR, Bastos A S, Guimarães M, Marcantonio Jr E. Experimental development of bisphosphonate-related osteonecrosis of the jaws in rodents. Int J Exp Pathol. 2013;94:65-73.

35. Bocanegra-Pérez MS, Vicente-Barrero M, Sosa-Henríquez M, RodríguezBocanegra E, Limiñana-Cañal JM, López-Márquez A, et al. Bone metabolism and clinical study of 44 patients with bisphosphonate-related osteonecrosis of the jaws. Med Oral Patol Oral Cir Bucal. 2012;17:e948-55.

36. Schilcher J, Koeppen V, Aspenberg P, Michaëlsson K. Risk of atypical femoral fracture during and after bisphosphonate use. N Engl J Med. 2014;371:974-6.

37. Meling T, Nawab A, Harboe K, Fosse L. Atypical femoral fractures in elderly women: a fracture registry-based cohort study. Bone Joint J. 2014:96-B(8):1035-40.

38. Blum L, Cummings K, Goulet JA, Perdue AM, Mauffrey C, Hake ME. Atypical femur fractures in patients receiving bisphosphonate therapy: etiology and management. Eur J Orthop Surg Traumatol. 2016;26:371-7.

39. Jilka RL, Weinstein RS, Bellido T, et al. Increased bone formation by prevention of osteoblast apoptosis with parathyroid hormone. J Clin Invest. 1999;104:439-46.

40. Dempster DW, Cosman F, Kurland ES, Zhou H, Nieves J, Woelfert L, et al. Effects of daily treatment with parathyroid hormone on bone microarchitecture and turnover in patients with osteoporosis: a paired biopsy study. J Bone Miner Res. 2001;16:1846-53.

41. Barnes GL, Kakar S, Vora S, Morgan EF, Gerstenfeld LC, Einhorn TA Stimulation of fracture-healing with systemic intermittent parathyroid hormone treatment. J Bone Joint Surg Am. 2008;90:120-7. 